\title{
Single-sperm analysis for recurrence risk assessment of spinal muscular atrophy
}

\author{
Philippe Burlet $^{1}$, Nadine Gigarel ${ }^{1}$, Maryse Magen ${ }^{1}$, Séverine Drunat ${ }^{2}$, Alexandra Benachi ${ }^{4}$, Laetitia Hesters ${ }^{3}$, \\ Arnold Munnich ${ }^{1}$, Jean-Paul Bonnefont ${ }^{1}$ and Julie Steffann ${ }^{1}$
}

With the detection of a homozygous deletion of the survival motor neuron 1 gene (SMN1), prenatal and preimplantation genetic diagnosis (PGD) for spinal muscular atrophy has become feasible and widely applied. The finding of a de novo rearrangement, resulting in the loss of the $S M N 1$ gene, reduces the recurrence risk from $25 \%$ to a lower percentage, the residual risk arising from recurrent de novo mutation or germline mosaicism. In a couple referred to our PGD center because their first child was affected with SMA, the male partner was shown to carry two SMN1 copies. An analysis of the SMN1 gene and two flanking markers was performed on 12 single spermatozoa, to determine whether the father carried a CIS duplication of the SMN1 gene on one chromosome and was a carrier, or if the deletion has occurred de novo. We showed that all spermatozoa that were carriers of the 'at-risk haplotype' were deleted for the SMN1 gene, confirming the carrier status of the father. We provide an original application of single germ cell studies to recessive disorders using coamplification of the gene and its linked markers. This efficient and easy procedure might be useful to elucidate complex genetic situations when samples from other family members are not available.

European Journal of Human Genetics (2010) 18, 505-508; doi:10.1038/ejhg.2009.198; published online 11 November 2009

Keywords: spinal muscular atrophy; prenatal diagnosis; preimplantation genetic diagnosis; single-sperm analysis; de novo mutation

\section{INTRODUCTION}

Spinal muscular atrophy is one of the most common autosomal recessive disorders, with a carrier frequency of $1 / 40-1 / 60 .{ }^{1}$ SMA is caused by the degeneration of motor neurons in the anterior horn of the spinal cord and has been classified into three types on the basis of clinical severity and age of onset: type I (Werdnig-Hoffmann disease, OMIM 253300), the most severe form; type II, an intermediate form (OMIM 253550); and type III (Kugelberg-Welander disease, OMIM 253400), the milder form. ${ }^{2,3}$

All three SMA types are associated with mutations in the survival motor neuron 1 gene (SMN1; OMIM 600354), located in the 5 q13 region, containing a $500-\mathrm{kb}$ duplication and inversion. ${ }^{4-8}$ SMN1 has a centromeric homolog, SMN2, which modulates the phenotype severity. ${ }^{9-10}$ SMN1 and 2 genes differ by only five nucleotides, only one of which is set in the coding sequence. ${ }^{11}$ Nucleotide variations in exons 7 and 8 are commonly used to discriminate between SMN1 and 2 genes, using a polymerase chain reaction/ restriction fragment length polymorphism assay. ${ }^{12}$ The majority of SMA patients (about 94\%) carry a homozygous deletion of at least exon 7 of the SMN1 gene, ${ }^{13}$ whereas the absence of the SMN2 gene has no clinical consequence in individuals carrying at least one SMN1 copy. SMN dosage gene analyses, which can determine SMN1 and SMN2 copy numbers, have been developed over the past 10 years, ${ }^{10,14-16}$ and show that (i) SMA patients who lack only one SMN1 gene carry allelic intragenic mutations (compound heterozygotes); ${ }^{15}$ and (ii) a CIS duplication of the SMN1 gene is found in $3-8 \%$ of putative carriers. ${ }^{16-18}$
A couple was referred to our clinic for preimplantation genetic diagnosis (PGD) because their first child was affected with SMA. DNA studies confirmed the diagnosis in the proband by demonstrating a homozygous deletion of the SMN1 exon 7 gene. SMN gene dosage analysis using a fluorescent multiplex PCR method ${ }^{16}$ indicated that the mother carried only one SMN1 copy, whereas the father was shown to carry two copies of SMN. Samples from the father's parents were not available, and there was no family history of SMA. Three situations might account for the father genotype (Figure 1): (i) a CIS duplication of the SMN1 gene on one chromosome with an absence of the SMN1 copy on the counterpart (' $2+0$ ' genotype); (ii) the occurence of a de novo deletion of a paternal chromosome carrying one SMN1 copy (one SMN1 copy on both chromosomes, called the genotype ' $1+1$ '); and (iii) a false paternity. The last hypothesis was ruled out by linkage analysis using microsatellites linked to the SMN1 gene (ie, D5S1988, ${ }^{19} \mathrm{D} 5 \mathrm{~S} 629,{ }^{20} \mathrm{C} 212,{ }^{8} \mathrm{C} 272,{ }^{8}$ and D5S637 ${ }^{20}$ ), which showed a paternal contribution to the child genotype for all tested markers (data not shown, available on request).

De novo deletions may occur in $2 \%$ or more of families with SMA, ${ }^{21,22}$ affecting preferentially the paternal chromosome. ${ }^{21}$ It has also been shown that a small proportion of parents carry the ' $2+0$ ' genotype, which is present in $\approx 3.2 \%$ of carriers. ${ }^{17}$ Using these data for Bayesian analysis as described in Ogino et al, ${ }^{1}$ we calculated a probability of $69 \%$ that the father was a ' $2+0$ ' carrier, and a probability of $31 \%$ that he was a ' $1+1$ ' noncarrier (Figure 1 ).

Distinguishing between these two hypotheses was important for reproductive decision making. The SMA recurrence risk in the

${ }^{1}$ Faculté de Médecine, Unité INSERM U781 Institut de Recherche Necker-Enfants Malades, service de génétique médicale, Hôpital Necker-Enfants Malades (Assistance Publique-Hôpitaux de Paris), Université Paris-Descartes, 75743 Paris Cedex 15, France; ${ }^{2}$ Service de Génétique Moléculaire, Hôpital Robert Debré (Assistance Publique-Hôpitaux de Paris), Paris, France; ${ }^{3}$ Maternité, Hôpital Necker-Enfants Malades, 75743 Paris Cedex 15, France; ${ }^{4}$ Service d'histo-embryologie cytogenetique, Hôpital Béclère, Clamart, France

Correspondence: Dr P Burlet, Hôpital Necker-Enfants Malades, 149 rue de Sèvres, 75743 Paris Cedex 15, France. Tel: +1 33144495164 ; Fax: +1 33171196420 ; E-mail: philippe.burlet@inserm.fr

Received 26 June 2009; revised 10 September 2009; accepted 11 September 2009; published online 11 November 2009 
Hypothesis1 (Bayesian probability 0.69)

The father has a " $2+0$ " genotype, he is a carrier of deletion of exon7 SMN1

Recurrence risk is $25 \%$

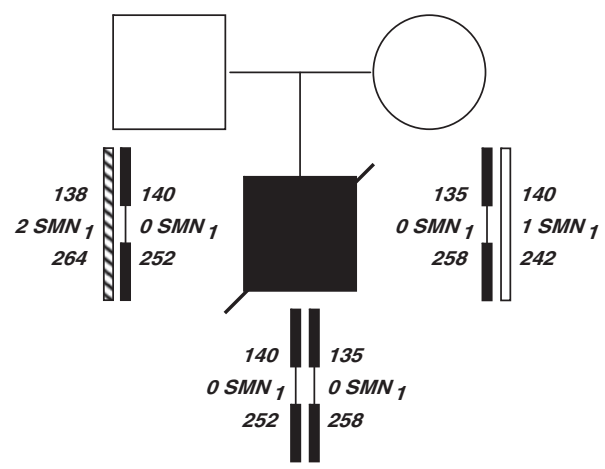

b

Hypothesis 2 (Bayesian probability 0.31 ) The mutation is «de novo", the father is " $1+1$ " Recurrence risk is low, coming from recurrent de novo mutation or germ-line mosaicism

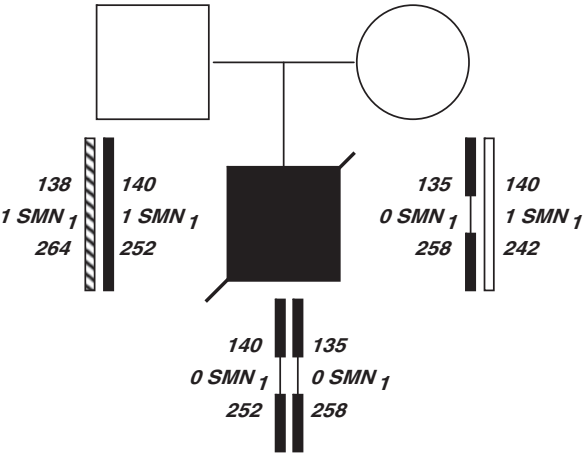

Figure 1 Pedigree of the family showing linkage analysis and the SMN1 copy number. Two hypotheses can account for the results of $S M N$ copy quantification and haplotyping from somatic cells of the nuclear family: (a) a CIS duplication of the SMN1 gene on one paternal chromosome with zero SMN1 copy on the counterpart; (b) occurrence of a de novo germ cell deletion on the paternal chromosome 5 . Black bars represented the affected maternal and 'at-risk' paternal alleles. Haplotypes at loci D5S629 (AFM265wf5) and D5S1988 (AFMa245we9) are shown from centromere to telomere, along with the results of the SMN1 gene dosage analysis.

offspring indeed decreased from $25 \%$ (genotype ' $2+0$ ') to a considerably lower figure in the second case (genotype ' $1+1$ '), reflected by the risk of germline mosaicism for the rearrangement in the father. Any application of the couple for PGD would have been prohibited in the last case. The French Law indeed authorizes PGD only when the genetic trait has previously been identified in the parents, preventing the use of PGD procedures for a risk of germline mosaicism.

In an attempt at clarifying this issue, we developed a strategy to determine the presence or absence of the SMN1 gene and haplotypes at the SMA locus from a single sperm cells.

\section{MATERIALS AND METHODS}

\section{Single-sperm cell isolation}

The technique was developed using single sperm cells obtained from freshly ejaculated sperm of the father. Spermatozoa were prepared using discontinuous gradient centrifugation. Separated sperms were rinsed and diluted in drops of PBS (Sigma Aldrich, Saint-Quentin Fallavier, France) supplemented with $0.1 \%$ polyvinyl alcohol (Sigma Aldrich) until single cells could be aspirated with a pulled glass micropipette in a clean laboratory. Single spermatozoa were transferred under visual control through an inverted microscope to PCR tubes containing lysis buffer $(3 \mu \mathrm{l}$ of $200 \mathrm{~mm} \mathrm{KOH}$, and $50 \mathrm{~mm} \mathrm{DTT}){ }^{23}$ Lysis was performed by heating at $65^{\circ} \mathrm{C}$ for $10 \mathrm{~min}$. A small volume of rinsing medium was transferred similarly and used as a negative template.

\section{Single-sperm PCR amplification}

The father was heterozygous for two linked [CA]n microsatellite markers (ie, D5S62920 and D5S1988 ${ }^{19}$ ), which are located on each side of the SMN1 gene (Figure 1). As these two markers showed a good amplification rate on single lymphocytes (data not shown), and because they flank the SMN1 gene, we selected them for sperm haplotype determination.

Polymerase chain reaction amplification conditions were similar to those used for blastomere analysis in SMA PGD, ${ }^{24}$ but amplification of D5S629 and D5S1988 was added to the detection of homozygous deletion of exon 7. Such amplification reactions enabled the mutant and wild-type alleles to be linked to individual haplotypes.
The multiplex 'outer' PCR reaction contained $3 \mu \mathrm{l}$ of lysis buffer (lysed spermatozoa or negative control), $2.5 \mu \mathrm{l}$ of primer mix $10 \times$ (containing each primer at $2 \mu \mathrm{m}$, Proligo, Evry, France), master mix $2 \times(12.5 \mu \mathrm{l}$, QIAGEN Multiplex PCR kit, QIAGEN S.A, Courtaboeuf, France), and double-distilled water up to a final volume of $25 \mu \mathrm{l}$.

For the 'inner' reaction, aliquots $(3 \mu \mathrm{l})$ from the multiplex 'outer' reaction were used as templates for individual PCR. Inner PCR primers for microsatellite amplification were $5^{\prime}$ fluorescently labeled with 6-FAM. 'Inner' amplification mixes contained primers $10 \times(2.5 \mu$ l, containing each primer at $2 \mu \mathrm{M}$, Proligo), master mix $2 \times(12.5 \mu$ l, QIAGEN Multiplex PCR kit), and double-distilled water up to $25 \mu \mathrm{l}$.

Polymerase chain reaction programs were similar for the outer and inner amplifications: $15 \mathrm{~min}$ denaturation at $95^{\circ} \mathrm{C}$, followed by 22 cycles (outer PCR) or 30 cycles (inner PCR) of $30 \mathrm{~s}$ at $94^{\circ} \mathrm{C}, 90 \mathrm{~s}$ at $60^{\circ} \mathrm{C}$, and $60 \mathrm{~s}$ at $72^{\circ} \mathrm{C}$, and terminated with a final extension of $30 \mathrm{~min}$ at $60^{\circ} \mathrm{C}$.

\section{Restriction enzyme digestion}

An aliquot of $10 \mu \mathrm{l}$ of the second round PCR product was incubated with 20 units of DraI (New England Biolabs, Ipswich, UK) at $37^{\circ} \mathrm{C}$ for $1 \mathrm{~h}$. PCR primers were designed to introduce a mismatch in order to create a second restriction site for the DraI enzyme in the SMN2 gene, allowing its differentiation with SMN1. Digested SMN1 amplicons yielded two fragments of 203 and $33 \mathrm{bp}$, and SMN2 yielded three fragments of 178, 33, and $25 \mathrm{bp}$, respectively. The products of enzymatic restriction digestion were resolved by electrophoresis on a $4 \%$ agarose gel (Nalgene, Rosckland, ME, USA) stained with ethidium bromide for $60 \mathrm{~min}$ at $90 \mathrm{~V}$, and visualized under UV light.

\section{Analysis of microsatellite markers}

Amplified 'inner' products $(1 \mu \mathrm{l})$ were added to a mix containing formamide ( $15 \mu \mathrm{l}$, genetic analysis grade, Applied Biosystems, Courtaboeuf, France) and ROX 400HD ( $0.3 \mu \mathrm{l}$, Applied Biosystems). After denaturation for $2 \mathrm{~min}$ at $95^{\circ} \mathrm{C}$, and fast cooling on ice, the products were electrophoresed in an automated genetic analyzer ABI 3130 (Applied Biosystems). Results were analyzed with Genescan and Genotyper softwares (Applied Biosystems). 


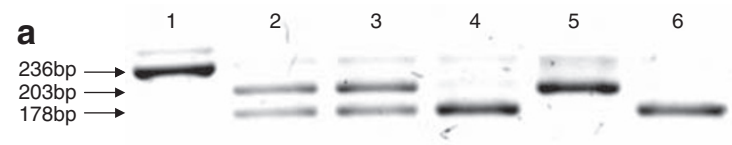

b

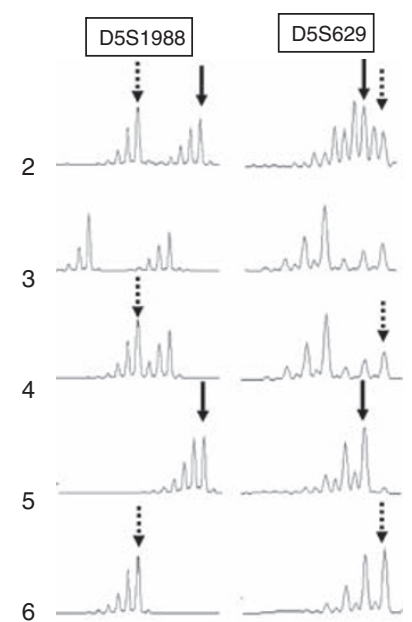

Figure 2 Analysis of SMN genes and detection of D5S1988 and D5S629 alleles on single spermatozoa. (a) Discrimination between SMN1 and SMN2 exon 7, using a PCR-Dral restriction approach $(236,203$, and $178 \mathrm{bp}$ depict undigested PCR products, SMN1 exon 7 and SMN2 exon 7, respectively). Lane 1: undigested PCR product. Lanes 2 and 3: proband's father and mother DNA are shown to carry both SMN1 and SMN2 exons 7. Lane 4: the proband carries SMN2 only. Lane 5: sperm 1 carrying only SMN1 copy. Lane 6: sperm 3 carrying only SMN2 copy. (b) Haplotype determination from a single sperm. Capillary electrophoregrams of amplified fluorescently labeled markers. Lanes $2-6$ are as in (a). Sperm 1 harbors the 'at-risk' allele (hatched arrow), whereas sperm 3 harbors the wild-type allele (full arrow).

\section{RESULTS AND DISCUSSION}

The proband and his parents were first haplotyped with D5S629 and D5S1988 markers, enabling the identification of the 'at-risk' paternal allele (Figure 1).

SMN1 exon 7 was successfully amplified in 7 out of 12 spermatozoa, whereas no amplification of SMN2 exon 7 was obtained in these cells (Figure 2 and Table 1). Conversely, a successful amplification of SMN2 exon 7 was obtained in 5 out of 12 spermatozoa, with no detection of SMN1 exon 7 (Table 1). No sperm carried both SMN1 and SMN2 genes, suggesting that $S M N 1$ and $S M N 2$ genes were carried on a different chromosome (Figure 2). Along the same line, no sperm carried two microsatellite alleles, supporting the fact that each tube contained only a single sperm cell.

Taking together (i) the results of the SMN1 and SMN2 copy number in the father somatic cells and (ii) the presence or absence of SMN1 and SMN2 in germ cells, we hypothesized that the father was a compound heterozygous at the somatic level, with one allele carrying a CIS duplication of the SMN1 gene and an SMN2 deletion, whereas the other chromosome carried zero SMN1 copy and one SMN2 gene (Figure 1a). A comparison of the proband's and spermatozoa's haplotypes achieved by polymorphic marker analysis confirmed these results by showing that 'SMN2 spermatozoa' carried the 'mutant haplotype', whereas 'SMN1 spermatozoa' had the wild-type one (Figure 2 and Table 1).

These data ruled out the hypothesis of a de novo mutation and therefore gave a $25 \%$ recurrence risk for each pregnancy. PGD for this
Table 1 Results of 12 single-sperm analysis for SMN1 mutation and linked markers

\begin{tabular}{lcccccccccccccc}
\hline \multicolumn{1}{c}{$\begin{array}{c}\text { Paternal } \\
\text { somatic } \\
\text { Markers }\end{array}$} & DNA & 1 & 2 & 3 & 4 & 5 & 6 & 7 & 8 & 9 & 10 & 11 & 12 \\
\hline D5S629 & $138 / 140^{\mathrm{a}}$ & 138 & 138 & 140 & - & 140 & 138 & 138 & - & 140 & - & 140 & 138 \\
SMN2 & $+(1$ copy $)$ & - & - & + & - & + & - & - & + & + & - & + & - \\
SMN1 & $+(2$ copy) & + & + & - & + & - & + & + & - & - & + & - & + \\
D5S1988 & $264 / 252^{a}$ & 264 & 264 & 252 & - & 252 & - & - & - & 252 & - & 252 & 262
\end{tabular}

'At-risk' haplotypes are shown in boldface type, and wild-type haplotypes in italics. The sign (-) means that no amplification product was obtained.

aNumbers represent marker allele length in base pairs (bp).

family thus became lawful. Amplification of SMN1 and SMN2 in sperm cells has been described for the purpose of diagnosing SMA carriers. ${ }^{25}$ In contrast to this approach, our method uses linked microsatellite markers in addition to the amplification of SMN1 and SMN2 to establish the sperm haplotype, and thereby decreases the number of single sperm cells that have to be tested. Amplification of SMN1 in both populations of spermatozoa (defined by different haplotypes) is indeed sufficient to define a 'non carrier' and to diagnose a 'de novo' deletion. In this case, our method additionally allows the detection of a germline mosaicism, provided that nonamplification of SMN1 in sperms carrying the at-risk allele is significantly more frequent than the 0.1 expected allele dropout rate. ${ }^{24}$

Single-sperm studies are useful for solving complex genetic situations. They have been used for the haplotype construction of de novo paternal mutations in dominant disorders, such as the neurofibromatosis type $1^{26}$ and Currarino syndrome, ${ }^{27}$ in order to reduce the misdiagnosis risk of PGD procedures. We provide here a further illustration of the crucial impact of single germ cell studies on genetics counseling in SMA, a recessive disorder.

\section{CONFLICT OF INTEREST}

The authors declare no conflict of interest.

1 Ogino S, Wilson RB: Genetic testing and risk assessment for spinal muscular atrophy (SMA). Hum Genet 2002; 111: 477-500.

2 Pearn J: Classification of spinal muscular atrophies. Lancet 1980; 1: 919-922.

3 Munsat TL: International SMA collaboration. Neuromuscular Disord 1991; 1: 81.

4 Melki J, Abdelhak S, Sheth P et al: Gene for chronic proximal spinal muscular atrophies maps to chromosome 5q. Nature 1990; 344: 767-768.

5 Brzustowicz LM, Lehner T, Castilla LH et al: Genetic mapping of chronic childhoodonset spinal muscular atrophy to chromosome 5q11.2-13.3. Nature 1990; 344: 540-541.

6 Gilliam TC, Brzustowicz LM, Castilla LH et al: Genetic homogeneity between acute and chronic forms of spinal muscular atrophy. Nature 1990; 345: 823-825.

7 Melki J, Sheth P, Abdelhak S et al: Mapping of acute (type I) spinal muscular atrophy to chromosome 5q12- q14. Lancet 1990; 336: 271-273.

8 Melki J, Lefebvre S, Burglen L et al: De novo and inherited deletions of the $5 \mathrm{q} 13$ region in spinal muscular atrophies. Science 1994; 264: 1474-1477.

9 Lefebvre S, Burlet P, Liu Q et al: Correlation between severity and SMN protein level in spinal muscular atrophy. Nat Genet 1997; 16: 265-269.

10 Feldkötter M, Schwarzer V, Wirth R et al: Quantitative analyses of SMN1 and SMN2 based on real-time lightCycler PCR: fast and highly reliable carrier testing and prediction of severity of spinal muscular atrophy. Am J Hum Genet 2002; 70: 358-368.

11 Bürglen L, Lefebvre S, Clermont 0 et al: Structure and organization of the human survival motor neurone (SMN) gene. Genomics 1996; 32: 479-482.

12 van der Steege G, Grootscholten PM, van der Vlies P et al: PCR-based DNA test to confirm clinical diagnosis of autosomal recessive spinal muscular atrophy. Lancet 1995; 345: 985-986.

13 Lefebvre S, Bürglen L, Reboullet S et al: Identification and characterization of a spinal muscular atrophy determining gene. Cell 1995; 80: 155-165. 
14 McAndrew PE, Parsons DW, Simard LR et al: Identification of proximal spinal muscular atrophy carriers and patients by analysis of SMNT and SMNC gene copy number. Am J Hum Genet 1997; 60: 1411-1422.

15 Wirth B, Herz M, Wetter A et al: Quantitative analysis of survival motor neuron copies: identification of subtle SMN1 mutations in patients with spinal muscular atrophy, genotype-phenotype correlation, and implications for genetic counseling. Am J Hum Genet 1999; 64: 1340-1356.

16 Saugier-Veber P, Drouot N, Lefebvre $S$ et al: Detection of heterozygous SMN1 deletions in SMA families using a simple fluorescent multiplex PCR method. J Med Genet 2001; 38: 240-243.

17 Chen KL, Wang YL, Rennert $\mathrm{H}$ et al: Duplications and de novo deletions of the SMNt gene demonstrated by fluorescence-based carrier testing for spinal muscular atrophy. Am J Med Genet 1999; 85: 463-469.

18 Ogino S, Wilson RB: SMN dosage analysis and risk assessment for spinal muscular atrophy. Am J Hum Genet 2002; 70: 1596-1598.

19 Dib C, Fauré S, Fizames $C$ et al: A comprehensive genetic map of the human genome based on 5,264 microsatellites. Nature 1996; 380: 152-154.

20 Gyapay G, Morissette J, Vignal A et al: The 1993-94 Généthon human genetic linkage map. Nat Genet 1994; 7: 246-339.
21 Wirth B, Schmidt T, Hahnen E et al: De novo rearrangements found in $2 \%$ of index patients with spinal muscular atrophy: mutational mechanisms, parental origin, mutation rate, and implications for genetic counseling. Am J Hum Genet 1997; 61: 1102-1111.

22 Zeesman S, Whelan DT, Carson N et al: Parents of children with spinal muscular atrophy are not obligate carriers: carrier testing is important for reproductive decisionmaking. Am J Med Genet 2002; 107: 247-249.

23 Cui XF, Li HH, Goradia TM et al: Single-sperm typing: determination of genetic distance between the $\mathrm{G}$ gamma-globin and parathyroid hormone loci by using the polymerase chain reaction and allele-specific oligomers. Proc Natl Acad Sci USA 1989; 86: 9389-9393.

24 Burlet P, Frydman N, Gigarel N et al: Improved single-cell protocol for preimplantation genetic diagnosis of spinal muscular atrophy. Fertil Steril 2005; 84: 734-739.

25 Yaron $\mathrm{Y}$, Cohen T, Mey-Raz N et al: Detection of spinal muscular atrophy carriers by nested polymerase chain reaction of single sperm cells. Genet Test 2006; 10: 18-23.

26 Altarescu G, Brooks B, Kaplan Y et al: Single-sperm analysis for haplotype construction of de-novo paternal mutations: application to PGD for neurofibromatosis type 1. Hum Reprod 2006; 21: 2047-2051.

27 Verlinsky Y, Rechitsky S, Schoolcraft W et al: Preimplantation diagnosis for homeobox gene HLXB9 mutation causing Currarino syndrome. Am J Med Genet A 2005; 134: 103-104. 